Bull. Austral. Math. Soc.

Vol. 49 (1994) [439-462]

\title{
ON SOME GEOMETRY ASSOCIATED WITH \\ A GENERALISED TODA LATTICE
}

\section{P.J. VASSILIOU}

We define the notion of Darboux integrability for linear second order partial differential operators,

$$
L=\partial_{x_{1} x_{2}}^{2}+\alpha \partial_{x_{1}}+\beta \partial_{x_{2}}+\gamma .
$$

We then build on certain geometric results of E. Vessiot related to the theory of Monge characteristics to show that the Darboux integrable operators $\mathrm{L}$ can be used to obtain a solution of the $A_{2}$ Toda field theory. This solution is parametrised by four arbitrary functions. The approach presented in this paper thereby represents an alternative means of linearising the $A_{2}$ Toda equations and may be contrasted with the known linearisation via the Lax pair.

\section{INTRODUCTION}

In an interesting paper [8], Leznov and Saveliev were able to obtain the zero curvature representation for the nonlinear system

$$
\frac{\partial^{2} u_{\alpha}}{\partial x_{1} \partial x_{2}}=\sum_{\beta=1}^{r} k_{\alpha \beta} \exp \left(u_{\beta}\right)
$$

for arbitrary real matrices $k=\left\{k_{\alpha \beta}\right\}_{\alpha, \beta=1}^{r}$. System (1.1) is encountered in a number of contexts in mathematical physics. For example, the cylindrically symmetric self dual Yang Mills fields in $\mathbb{R}^{4}$ are described by (1.1) when $k$ coincides with the Cartan matrix of the relevant gauge group. If $k$ coincides with the Cartan matrix of the series $A_{r}$ of simple Lie algebras then (1.1) is equivalent to the generalised Toda chain with free end points. Leznov and Saveliev discovered, using the theory of Lie algebra representations, that in these special cases, that is, when $k$ is the Cartan matrix of a simple Lie algebra of rank $r$, they could obtain an explicit solution of (1.1) involving $2 r$ arbitrary functions which they called the general solution. In their paper they posed the question: "why is

Received 12th July, 1993

The results reported in this paper were obtained while 1 was a visitor at the Centre de Recherches Matematiques, Universite de Montreal. I would like to thank the Centre and its staff for hospitality and support and for providing an excellent atmosphere for research. I would also like to thank Professors M. Grundland, J. Harnad, and N. Kamran for helpful comments and discussions which sharpened my views of several of the results here reported.

Copyright Clearance Centre, Inc. Serial-fee code: 0004-9729/94 \$A2.00+0.00. 
system (1.1) explicitly integrable in just these cases ?" The purpose of the present paper is to show that when $k$ is the Cartan matrix of the Lie algebra $A_{2}$, the general solution is strongly linked to the integrability in the Darboux sense of the linear hyperbolic partial differential equation

where

$$
\begin{gathered}
\frac{\partial^{2} w}{\partial x_{1} \partial x_{2}}+\alpha \frac{\partial w}{\partial x_{1}}+\beta \frac{\partial w}{\partial x_{2}}+\gamma w=0 \\
\alpha, \beta, \gamma \in C^{\infty}\left(\mathbb{R}^{2}, \mathbb{R}\right) .
\end{gathered}
$$

The main idea is that (1.2) is Darboux integrable if and only if a variant of the $A_{2}$ Toda equations are satisfied. This fact together with a fundamental result of Vessiot [12] on the classification of Darboux integrable semilinear hyperbolic partial differential equations allows one to construct an explicit solution in finite terms of four arbitrary functions each of one variable.

This result in fact bears out general remarks made by Hermann [7] and illustrates a paradigm concerning integrable nonlinear partial differential equations, namely that every integrable nonlinear partial differential equation is associated with a "linear problem" whose "integrability condition" is the nonlinear partial differential equation under study. In the usual discussions of completely integrable systems the linear problem of interest is derived from the Lax pair. Here we point out another type of linear problem, namely the equation (1.2) while the notion of "integrability condition" is replaced in this context by the notion of Darboux integrability.

Of course the idea of Darboux integrability which is to be described is a classical one and when it is applied to equation (1.2) one discovers that it is strongly linked to yet another classical notion, that of a Laplace Transformation (not to be confused with a Laplace transform). This topic together with its relevance to various nonlinear partial differential equation now known to be completely integrable was first discussed by Darboux in the second volume of his treatise [2] and effectively reviewed in Hermann [7, Chapter IX] More recently, the connection between the Laplace transformation and various Toda lattices has been discussed in Weiss [13]. In a sense the results of this paper are complementary to those presented in Weiss and it would be interesting to make the connections precise.

By now many papers have been written on the Toda equations and no attempt will be made here to place the results of this paper within the spectrum of known results. We shall be content here to show what the classical notions of Darboux integrability and Laplace transformations have to do with the $A_{2}$ Toda field theory.

\section{LAPLACE TRANSFORMATION}

In this section we briefly review some notions associated with the idea of a Laplace 
transformation of equation (1.2). For related material on this topic the reader is referred to Hermann [7].

Let $\mathcal{D}$ denote the set of second order linear partial differential operators of the form

where

$$
L=\partial_{x_{1} x_{2}}^{2}+\alpha \partial_{x_{1}}+\beta \partial_{x_{2}}+\gamma
$$

$$
\alpha, \beta, \gamma \in C^{\infty}\left(\mathbb{R}^{2}, \mathbb{R}\right) \text {. }
$$

There is an interesting Lie pseudogroup of transformations $\Psi$ which which acts on $\mathcal{D}:$

$$
\begin{gathered}
\Psi: G \times \mathcal{D} \rightarrow \mathcal{D}, \quad G=C^{\infty}\left(\mathbb{R}^{2}, \mathbb{R}\right), \\
\text { defined by } \\
\Psi(\lambda, L)=T_{\lambda}(L)=\lambda^{-1} L \lambda, \quad \lambda \in G, L \in \mathcal{D},
\end{gathered}
$$

and where the group operation in $G$ is point multiplication of functions. A straightforward computation then gives

$$
\text { (2.1) } \begin{aligned}
T_{\lambda}(L)=\partial_{x_{1} x_{2}}^{2}+\left(\alpha+\lambda^{-1} \partial_{x_{2}} \lambda\right) & \partial_{x_{1}}+\left(\beta+\lambda^{-1} \partial_{x_{1}} \lambda\right) \partial_{x_{2}} \\
& +\left(\lambda^{-1} \partial_{x_{1} x_{2}}^{2} \lambda+\alpha \lambda^{-1} \partial_{x_{1}} \lambda+\beta \lambda^{-1} \partial_{x_{2}} \lambda+\gamma\right)
\end{aligned}
$$

Next, consider the set of all maps $\mathcal{D} \rightarrow C^{\infty}\left(\mathbb{R}^{2}, \mathbb{R}\right)$. Denote this set by $\mathcal{F}$. From the above $G$-action on $\mathcal{D}$, there is an induced action on $\mathcal{F}$ defined by

$$
\tau_{\lambda}(f)(L)=f\left(T_{\lambda} L\right), \quad f \in \mathcal{F}, \lambda \in G .
$$

It is now of interest to enquire about the invariants of this $G$-action on $\mathcal{F}$. That is, we seek functions $f: \mathcal{D} \rightarrow C^{\infty}\left(\mathbb{R}^{2}\right)$ such that

$$
\tau_{\lambda}(f)(L)=f(L), \quad \lambda \in G, L \in \mathcal{D} \text {. }
$$

Classically, two such invariants are known:

$$
\begin{aligned}
& h(L)=\partial_{x_{1}} \alpha+\alpha \beta-\gamma, \\
& k(L)=\partial_{x_{2}} \beta+\alpha \beta-\gamma .
\end{aligned}
$$

It is straightforward to show from equation (2.1) that

$$
\tau_{\lambda}(h)(L)=h\left(T_{\lambda} L\right)=h\left(\lambda^{-1} L \lambda\right)=h(L), \quad \text { for all } \quad \lambda \in G, L \in \mathcal{D}
$$

and similarly for $k$. This establishes $h$ and $k$ as differential invariants in $\mathcal{F}$ known classically as the Laplace invariants of $L$. The Laplace invariants will play a key role in our subsequent considerations. 


\section{The Laplace transformation as a Backlund MaP.}

We are now in a position to introduce the Laplace transformation and to establish a number of properties which will later prove useful. Suppose we consider a set of partial differential equations

$$
\begin{aligned}
u_{1}-\partial_{x_{2}} u-\alpha u & =0 \\
\partial_{x_{1}} u_{1}+\beta u_{1}-\zeta u & =0
\end{aligned}
$$

for two unknown functions $u, u_{1} \in C^{\infty}\left(\mathbb{R}^{2}, \mathbb{R}\right)$, where $\alpha, \beta, \zeta$ are given real-valued functions of $x_{1}$ and $x_{2}$. We ask the question: what should the function $\zeta\left(x_{1}, x_{2}\right)$ be in order that the function $u\left(x_{1}, x_{2}\right)$ satisfy the partial differential equation $L u=0$ ? The answer is $\zeta=h(L)$.

Matters being so, one finds that the partial differential equation satisfied by the function $u_{1}\left(x_{1}, x_{2}\right)$ is $L_{1} u_{1}=0$, where,

$$
L_{1}=\partial_{x_{1} x_{2}}^{2}+\left(\alpha-\partial_{x_{2}} \ln |h(L)|\right) \partial_{x_{1}}+\beta \partial_{x_{2}}+\left(\gamma-\partial_{x_{1}} \alpha+\partial_{x_{2}} \beta-\beta \partial_{x_{2}} \ln |h(L)|\right)
$$

Clearly, then, equations (2.4) and (2.5) define a nonauto Backlund transformation relating the solutions of $L \phi=0$ to those of $L_{1} \phi=0$.

There is one further nonauto Backlund transformation that may be written down analogous to (2.4) and (2.5) but involving the other Laplace invariant, namely

$$
\begin{aligned}
& u_{-1}-\partial_{x_{1}} u-\beta u=0 \\
& u_{-1}-k(L) u-\alpha u_{-1}
\end{aligned}
$$

Once more, by eliminating the unknown function $u_{-1}$, one finds that $u$ satisfies the partial differential equation $L u=0$ while $u_{-1}$ satisfies $L_{-1} u_{-1}=0$ where,

$$
L_{-1}=\partial_{x_{1} x_{2}}^{2}+\left(\beta-\partial_{x_{1}} \ln |k(L)|\right) \partial_{x_{2}}+\alpha \partial_{x_{1}}+\left(\gamma-\partial_{x_{2}} \beta+\partial_{x_{1}} \alpha-\alpha \partial_{x_{1}} \ln |k(L)|\right)
$$

as may easily be verified. The operators $L_{1}$ and $L_{-1} \in \mathcal{D}$ are known as the Laplace transformations of $L \in \mathcal{D}$. The notation used here in fact goes back to Darboux and is a little misleading because it gives the impression that the Laplace transformations $\mathcal{L}_{1}: L \rightarrow L_{1}$ and $\mathcal{L}_{-1}: L \rightarrow L_{-1}$ are inverses, which they are not. However, if $\widehat{\mathcal{D}}$ denotes the space of orbits in $\mathcal{D}$ under $G$ then it may be shown that $\mathcal{L}_{1}$ and $\mathcal{L}_{-1}$ map $G$-orbits to $G$-orbits, that is, if $\Delta_{1}, L_{1}$ are the images of $\Delta, L \in \mathcal{D}$ respectively, then if $\Delta$ and $L$ share an orbit, there exists $\lambda, \mu \in G$ such that the diagram

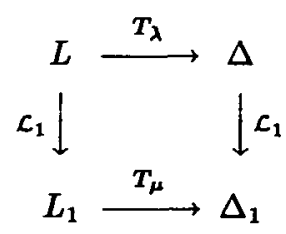


commutes. Similarly for $\mathcal{L}_{-1}$. Hence $\mathcal{L}_{1}$ and $\mathcal{L}_{-1}$ pass to the quotient to act on the orbit space $\widehat{\mathcal{D}}$. It is then easily shown that

and

$$
\begin{aligned}
& \mathcal{L}_{-1} \circ \mathcal{L}_{1}(L)=k(L) L k(L)^{-1} \\
& \mathcal{L}_{1} \circ \mathcal{L}_{-1}(L)=h(L) L h(L)^{-1}
\end{aligned}
$$

which proves that the Laplace transformations $\mathcal{L}_{1}$ and $\mathcal{L}_{-1}$ are, when acting on $\widehat{\mathcal{D}}$, inverses of each other. These facts are pointed out by Hermann [7] who also reviews the way in which Darboux used them to study classes of nonlinear Kein-Gordon equations now known to be completely integrable. All these results may be compared with those of Weiss [13]

We end this section on the Laplace transformation by recording the Laplace invariants of $L_{1}$ and $L_{-1}$ since they will make an appearance in later computations :

$$
\begin{gathered}
h\left(L_{1}\right)=2 h(L)-k(L)-\partial_{x_{1} x_{2}}^{2} \ln |h(L)|, \\
k\left(L_{1}\right)=h(L), \\
h\left(L_{-1}\right)=k(L), \\
k\left(L_{-1}\right)=2 k(L)-h(L)-\partial_{x_{1} x_{2}}^{2} \ln |k(L)| .
\end{gathered}
$$

\section{DARBOUX INTEgRabILITY OF NONLINEAR HYPERBOLIC PARTIAL DIFFERENTIAL EQUATIONS IN THE PLANE}

The field of integrability of nonlinear partial differential equations is still relatively young having begun, properly speaking, in 1967 when Gardner, Greene, Kruskal and Miura published the solution of the initial value problem of the Korteweg de Vries equation by the inverse scattering transform.

In the intervening years, it has been realised that an enormous inheritance has been left to the current generation by the 19th century in the field of integrability; particularly by geometers like Lie, Darboux and Goursat to mention but a few. As a consequence, a great deal of work needs to be done to codify and clarify this body of results and particularly its later developments by Cartan and Vessiot and to work out its consequences and limitations for integrability and solvabilty questions associated with differential equations. Already in this direction a number of workers have made important contributions which have covered topics such as Lie equations, symmetry groups, prolongation structures, differential Galois theory to mention but a few.

In this section we wish to review briefly another classical construction due to Darboux and extending well known results of Monge. Darboux's construction is based on the notion of Monge characteristics associated with any nonelliptic partial differential equation and which generalises the notion of Cauchy characteristics. 
The theory of characteristics is most clearly expressed by making use of Cartan's geometric results [1]. In the hyperbolic case, this has been most lucidly expounded in Gardner and Kamran [4] where aspects of the Cartan equivalence problem for hyperbolic partial differential equations in two independent variables is also covered. In this paper we shall not present a full geometric exposition of Darboux's idea and the reader is referred to Vassiliou [11] for this. We shall instead be content here to present enough details in order to prepare the reader for an application of Darboux's construction to the $A_{2}$ Toda field theory.

Consider the second order quasilinear partial differential equation

$$
A \frac{\partial^{2} u}{\partial x_{1}^{2}}+2 H \frac{\partial^{2} u}{\partial x_{1} \partial x_{2}}+B \frac{\partial^{2} u}{\partial x_{2}^{2}}=0
$$

where $A, H, B$ and $C$ are $C^{1}$ functions of the variables $x_{1}, x_{2}, u, \frac{\partial u}{\partial x_{1}}$ and $\frac{\partial u}{\partial x_{2}}$.

It has been known since the time of Monge that the equation (3.1) may be associated with a pair of modules of differential 1-forms defined on $J^{1}\left(\mathbb{R}^{2}, \mathbb{R}\right)$, the manifold of 1 -jets of maps $\mathbb{R}^{2} \rightarrow \mathbb{R}$. Let us denote these modules by ${ }_{1} \widetilde{\Omega}^{(1)}$ and ${ }_{2} \widetilde{\Omega}^{(2)}$ and let $\omega=H+\sqrt{H^{2}-A B}$. Then whenever $H^{2}-A B \geq 0$ we have that ${ }_{1} \widetilde{\Omega}^{(1)}$ and ${ }_{2} \widetilde{\Omega}^{(1)}$ are generated as follows

$$
\begin{aligned}
& { }_{1} \widetilde{\Omega}^{(1)}:\left\{\theta_{1}^{1}=\omega d x_{1}-A d x_{2}, \theta_{2}^{1}=\omega d z_{1}+B d z_{2}-C d x_{2}, \theta_{3}^{1}=d z-z_{1} d x_{1}-z_{2} d x_{2}\right\} \\
& { }_{2} \widetilde{\Omega}^{(1)}:\left\{\theta_{1}^{2}=B d x_{1}-\omega d x_{2}, \theta_{2}^{2}=A d z_{1}+\omega d z_{2}-C d x_{1}, \theta_{3}^{2}=d z-z_{1} d x_{1}-z_{2} d x_{2}\right\}
\end{aligned}
$$

where here as elsewhere curly brackets denote module closure over (in this case) $C^{\infty}\left(J^{1}\left(\mathbb{R}^{2}, \mathbb{R}\right), \mathbb{R}\right)$.

I will not go into the genisis of these modules which were first obtained by Monge in order to integrate equations like (3.1). Neither will $I$ in this paper attempt to connect the modules ${ }_{1} \widetilde{\Omega}^{(1)}$ and ${ }_{2} \widetilde{\Omega}^{(1)}$ to the work of Cartan as expounded by Gardner and Kamran [4]. Suffice it to say for the present that the maximal dimension regular integral submanifolds of these two modules are one in each case and represent what are classically known as the (order 1) Monge characterisics of the partial differential equation (3.1). For our immediate purposes, the significance of ${ }_{1} \widetilde{\Omega}^{(1)}$ and ${ }_{2} \tilde{\Omega}^{(1)}$ is the following.

TheOREM 3.1. Suppose there exists functions $\lambda_{1}, \lambda_{2}, \lambda_{3}: J^{1}\left(\mathbb{R}^{2}, \mathbb{R}\right) \rightarrow \mathbb{R}$ such that

$$
\lambda_{1} \theta_{1}^{i}+\lambda_{2} \theta_{2}^{i}+\lambda_{3} \theta_{3}^{i}=d F
$$

for $i=1$ or 2 and for some function $F: J^{1}\left(\mathbb{R}^{2}, \mathbb{R}\right) \rightarrow \mathbb{R}$. If $f \in C^{2}\left(\mathbb{R}^{2}, \mathbb{R}\right)$ is such that

$$
\left(j^{1} f\right)^{\star} F=0
$$


then $f$ is a solution of the second order partial differential equation (3.1) where $j^{1} f$ : $\mathbb{R}^{2} \rightarrow J^{1}\left(\mathbb{R}^{2}, \mathbb{R}\right)$ is the 1-graph of $f$.

PROOF: See Vassiliou [11].

REMARK. $F$, when it exists, is called a first integral of (3.1). Evidently this is a very advantageous situation since it means that solutions of a second order partial differential equation (3.1) may be obtained by solving a first order partial differential equation (3.2). Because of the existence of Cauchy vector fields in the latter case the integration of (3.1) is thereby reduced to ordinary differential equations.

An equation (3.1) for which $F$ exists (as in Theorem 3.1) for $i=1$ or 2 will be called Monge integrable or, alternatively, Darboux integrable at order 1. If (3.1) is Monge integrable and $i=1$, it will be called Monge integrable on system 1 ; if $i=2$ then (3.1) will be called Monge integrable on system 2. We wish to use all these results to study equations like (1.2). The proof of the following can be found in Grundland and Vassiliou [5]

THEOREM 3.2. The linear hyperbolic partial differential equation

$$
\frac{\partial^{2} u}{\partial x_{1} \partial x_{2}}+\alpha\left(x_{1}, x_{2}\right) \frac{\partial u}{\partial x_{1}}+\beta\left(x_{1}, x_{2}\right) \frac{\partial u}{\partial x_{2}}+\gamma\left(x_{1}, x_{2}\right) u=0
$$

is Monge integrable on system 1 if and only if

$$
h=\frac{\partial \alpha}{\partial x_{1}}+\alpha \beta-\gamma=0 .
$$

and Monge integrable on system 2 if and only if

$$
k=\frac{\partial \beta}{\partial x_{2}}+\alpha \beta-\gamma=0 .
$$

There can be only one such functionally independent first integral.

A remarkable fact about the modules ${ }_{1} \widetilde{\Omega}^{(1)}$ and ${ }_{2} \widetilde{\Omega}^{(1)}$ and Theorem 3.1 is that the whole construction can be lifted to higher order jet bundles where new results are to be found. This lift is known as Darboux's method. We shall formulate the results for the equation

$$
\frac{\partial^{2} u}{\partial x_{1} \partial x_{2}}=f\left(x_{1}, x_{2}, u, \frac{\partial u}{\partial x_{1}}, \frac{\partial u}{\partial x_{2}}\right)
$$

however we note that this restriction is unnecessary and Darboux's method applies to a larger set of equations than that represented by (3.3). It is possible to describe Darboux's method by defining an appropriate pair of differential 1-forms on the $k^{t h}$ 
order jet bundle. For the purposes of later calculation however we shall instead express everything in terms of vector fields.

Let $\Delta_{k}$ denote the subset of $J^{k}\left(\mathbb{R}^{2}, \mathbb{R}\right)$ determined by the $(k-2)$ prolongation of equation (3.3). Thus $\Delta_{3} \subset J^{3}\left(\mathbb{R}^{2}, \mathbb{R}\right)$ is defined by

$$
\begin{gathered}
z_{12}=f\left(x_{1}, x_{2}, z, z_{1}, z_{2}\right), z_{112}=\frac{\partial f}{\partial x_{1}}+z_{1} \frac{\partial f}{\partial z}+z_{11} \frac{\partial f}{\partial z_{1}}+z_{12} \frac{\partial f}{\partial z_{2}} \\
z_{122}=\frac{\partial f}{\partial x_{2}}+z_{2} \frac{\partial f}{\partial z}+z_{12} \frac{\partial f}{\partial z_{1}}+z_{22} \frac{\partial f}{\partial z_{2}}
\end{gathered}
$$

Let $D_{1}^{(k)}$ and $D_{2}^{(k)}$ be total differential operators in $J^{k}\left(\mathbb{R}^{2}, \mathbb{R}\right)$ restricted to the subset $\Delta_{k}$.

DEFINITION 3.1: The vector field systems

$$
\begin{aligned}
& { }_{1} \Omega^{(k)}(f):\left\{D_{1}^{(k)}+\left(\left(D_{2}^{(k)}\right)^{k-1} f\right) \frac{\partial}{\partial z_{22 \ldots 2}}, \frac{\partial}{\partial z_{11 \ldots 1}}\right\} \\
& { }_{2} \Omega^{(k)}(f):\left\{D_{2}^{(k)}+\left(\left(D_{1}^{(k)}\right)^{k-1} f\right) \frac{\partial}{\partial z_{11 \ldots 1}}, \frac{\partial}{\partial z_{22 \ldots 2}}\right\}
\end{aligned}
$$

which are modules of vector fields on $\Delta_{k}$ will be called the $k^{\text {th }}$ order Monge characteristic vector field systems associated with equation (3.3).

REMARK. The vector field systems ${ }_{1} \Omega^{(k)}$ and ${ }_{2} \Omega^{(k)}$ generalise ${ }_{1} \widetilde{\Omega}^{(1)}$ and ${ }_{2} \widetilde{\Omega}^{(1)}$ in that by setting $k=1$ in ${ }_{1} \Omega^{(k)}$ and ${ }_{2} \Omega^{(k)}$ and computing their duals we obtain precisely the modules $\widetilde{\Omega}^{(1)}$ and ${ }_{2} \widetilde{\Omega}^{(1)}$. It may be checked that the vector field systems of Definition 3.1 each have genus $g=1$. Furthermore, their 1 -dimensional integral submanifolds are classically known as the $k^{\text {th }}$ order Monge characteristics. Full details will be found in Vassiliou [11]

Our interest for the present is the generalisation of Theorem 3.1 which is the following.

ThEOREM 3.3. Let $\psi\left(x_{1}, x_{2}\right)$ be any $C^{k+1}$ solution of

$$
\frac{\partial^{2} u}{\partial x_{1} \partial x_{2}}=f\left(x_{1}, x_{2}, u, \frac{\partial u}{\partial x_{1}}, \frac{\partial u}{\partial x_{2}}\right)
$$

Suppose $\pi: \Delta_{k} \rightarrow \mathbb{R}$ is an invariant of ${ }_{i} \Omega^{(k)}$, for $i=1,2$. Then

$$
\frac{\partial}{\partial x_{i}}\left(\pi \circ j^{k} \psi\right)=0, \quad i=1,2
$$


where $j^{k} \psi$ is the $k$-graph of $\psi$.

Proof: For each $i=1,2$, let $M_{1}^{i}$ and $M_{2}^{i}$ be the two vector fields generating ${ }_{i} \Omega^{(k)}$. Then if $\psi$ is the stated solution we have

$$
\left(j^{k} \psi\right)_{\star}\left(\frac{\partial}{\partial x_{i}}\right)(\pi)=\frac{\partial}{\partial x_{i}}\left(\pi \circ j^{k} \psi\right)=\left(M_{1}^{i} \pi\right) \circ j^{k} \psi+\frac{\partial^{k+1} \psi}{\partial x_{i}^{k+1}}\left(M_{2}^{i} \pi\right) \circ j^{k} \psi=0
$$

by definition of $\pi$.

Theorem 3.3 makes clear the fact that from equation (3.3) we have constructed a compatible system of partial differential equations, assuming that we are in fact able to construct the invariants $\pi_{1}$ of $\Omega_{1} \Omega^{(k)}$ and $\pi_{2}$ of ${ }_{2} \Omega^{(k)}$. These equations are as follows.

$$
\begin{gathered}
\frac{\partial^{2} \psi}{\partial x_{1} \partial x_{2}}=f\left(x_{1}, x_{2}, \psi, \frac{\partial \psi}{\partial x_{1}}, \frac{\partial \psi}{\partial x_{2}}\right), \\
\frac{\partial}{\partial x_{1}}\left(\pi_{1} \circ j^{k} \psi\right)=0 \\
\frac{\partial}{\partial x_{2}}\left(\pi_{2} \circ j^{k} \psi\right)=0 .
\end{gathered}
$$

This system is, by Theorem 3.3, guaranteed to be compatible whenever equation (3.4) has nontrivial $C^{k+1}$ solutions. At this point it may be argued that system (3.4) to (3.6) is a worse problem than solving (3.4) by itself. In the first place the system is over determined and the possibility of nontrivial integrability conditions is not excluded in fact by Theorem 3.3. In the second place, the order of system (3.4) to (3.6) is in general higher than 2 . So what advantage is provided by this Theorem?

A close inspection of equations (3.5) and (3.6) shows in fact that even though $\psi$ is a function of the two variables $x_{1}$ and $x_{2}$, the equation (3.5) contains no differentiations with respect to $x_{1}$ while (3.6) contains no differentiations with respect to $x_{2}$. This is because $x_{1}$ and $x_{2}$ are themselves invariants of ${ }_{2} \Omega^{(k)}$ and $\Omega_{1}^{(k)}$ respectively and hence are what are commonly referred to as characteristic variables. The effect of this is that equations (3.5) and (3.6) are not really partial differential equations at all but parametrised families of ordinary differential equations. In order to integrate equation (3.4) we turn Theorem 3.3 on its head and study instead of (3.4) either or both of equations (3.5) and (3.6). If we succeed in solving these ordinary differential equations then by Theorem 3.3, we also obtain solutions of the original second order partial differential equation (3.4). This makes clear the very interesting fact that even though second order partial differential equations have no Cauchy vectors associated to them in general, if their Monge fields carry sufficient invariants, the construction of their general solutions can be reduced to solving ordinary differential equations. To see 
explicit examples of this the reader is referred Grundland and Vassiliou $[5,6]$ and to Vassiliou [11].

In order to study the $A_{2}$ Toda field theory we need to generalise Theorem 3.2 to the second order jet bundle $J^{2}\left(\mathbb{R}^{2}, \mathbb{R}\right)$ and to study the integrability via Darboux's method of equation (1.2).

We begin by letting $\Gamma_{k}$ denote the set of direct sums of modules ${ }_{1} \Omega^{(k)}(f) \oplus_{2} \Omega^{(k)}(f)$ as $f$ ranges over all partial differential equations (3.3). Let $\tilde{\mu}$ be the map which assigns to each $f$ its direct sum of Monge characteristic modules in $\Gamma_{k}$. Let $\mu$ be the restriction of $\tilde{\mu}$ to the partial differential equations $L u=0, L \in \mathcal{D}$. Specifically, $\mu: \mathcal{D} \rightarrow \Gamma_{2}$ is defined by $\mu(L)={ }_{1} \Omega^{(2)}(L) \oplus{ }_{2} \Omega^{(2)}(L)$, where ${ }_{i} \Omega^{(2)}(L), i=1,2$, are the Monge characteristic vector field systems associated to the partial differential equation $L u=0$. Let $\Gamma_{2}(2,2)$ denote the subset of $\Gamma_{2}$ for which $i \Omega^{(2)}(L), i=1,2$, each possess precisely two functionally independent invariants and let $\gamma_{2}(2,2)$ be the restriction of $\Gamma_{2}(2,2)$ to the partial differential equation $L u=0, L \in \mathcal{D}$.

A partial differential equation (3.3) will be called (2,2)-Darboux integrable at order 2 if $\tilde{\mu}(f) \in \Gamma_{2}(2,2)$. Similarly an operator $L \in \mathcal{D}$ will be called $(2,2)$-Darboux integrable at order 2 or, simply, (2,2)-Darboux integrable if $\mu(L) \in \gamma_{2}(2,2)$. The following theorem characterises the $(2,2)$-Darboux integrable operators in $\mathcal{D}$.

ThEOREM 3.4. Let

$$
L=\partial_{x_{1} x_{2}}+\alpha \partial_{x_{1}}+\beta \partial_{x_{2}}+\gamma
$$

be a linear second order partial differential operator belonging to $\mathcal{D}$. Then the partial differential equation

$$
L u=0
$$

has precisely two invariants on system ${ }_{1} \Omega^{(2)}$ if and only if $h(L)$ and $k(L)$ satisfy

$$
\frac{\partial^{2}}{\partial x_{1} \partial x_{2}} \ln |h(L)|=2 h(L)-k(L), \quad h(L) \neq 0
$$

and precisely two invariants on system ${ }_{2} \Omega^{(2)}$ if and only if

$$
\frac{\partial^{2}}{\partial x_{1} \partial x_{2}} \ln |k(L)|=-h(L)+2 k(L), \quad k(L) \neq 0 .
$$

Proof: In Grundland and Vassiliou [5], it is shown that $L u=0$ has precisely two invariants on system ${ }_{1} \Omega^{(2)}$ if and only if the determinant of the matrix

$$
\left(\begin{array}{cc}
h & K \\
\frac{\partial h}{\partial x_{1}}+\alpha h & \frac{\partial K}{\partial x_{1}}+h B+\alpha K
\end{array}\right)
$$


is zero while $h(L) \neq 0$, where

$$
\begin{aligned}
& K=\frac{\partial A}{\partial x_{1}}+\alpha B+\beta A-C, A=\frac{\partial \alpha}{\partial x_{2}}+\alpha^{2}, \\
& B=\frac{\partial \beta}{\partial x_{2}}-\alpha \beta+\gamma, C=\frac{\partial \gamma}{\partial x_{2}}-\alpha \gamma .
\end{aligned}
$$

Calculation in fact shows that

$$
K=\frac{\partial h}{\partial x_{1}}-2 \alpha h .
$$

Hence the determinantal condition becomes

$$
\begin{aligned}
0= & \frac{\partial K}{\partial x_{1}}+h B-\frac{K}{h} \frac{\partial h}{\partial x_{1}}=\frac{\partial^{2} h}{\partial x_{1} \partial x_{2}}-2 \alpha \frac{\partial h}{\partial x_{1}}-2 h \frac{\partial \alpha}{\partial x_{1}} \\
& \quad+h(k-2 \alpha \beta+2 \gamma)-h^{-1}\left(\frac{\partial h}{\partial x_{2}}-2 \alpha h\right) \frac{\partial h}{\partial x_{1}} \\
= & \frac{\partial^{2} h}{\partial x_{1} \partial x_{2}}-2 h\left(\frac{\partial \alpha}{\partial x_{1}}+\alpha \beta-\gamma\right)-h^{-1} \frac{\partial h}{\partial x_{1}} \frac{\partial h}{\partial x_{2}}+h k \\
= & h^{-1} \frac{\partial^{2} h}{\partial x_{1} \partial x_{2}}-h^{-2} \frac{\partial h}{\partial x_{1}} \frac{\partial h}{\partial x_{2}}-2 h+k \\
= & \frac{\partial^{2}}{\partial x_{1} \partial x_{2}} \ln |h|-2 h+k
\end{aligned}
$$

which is condition (3.7).

A similar calculation associated with the module ${ }_{2} \Omega^{(2)}$ leads to the equation (3.8). Let us note that the equations (3.7) and (3.8) correspond precisely to the vanishing of the Laplace invariants $h\left(L_{1}\right)$ and $k\left(L_{-1}\right)$; see Section 2.

It follows from Theorem 3.4 that $\mu(L) \in \gamma_{2}(2,2)$ if and only if $h(L)$ and $k(L)$ satisfy the coupled system (3.7) and (3.8). In the next section we shall show how the class $\mu^{-1}\left(\gamma_{2}(2,2)\right)$ can be explicitly constructed. Indeed we give a parametrisation of $\mu^{-1}\left(\gamma_{2}(2,2)\right)$ in terms of four arbitrary functions. This parametrisation was first obtained by Vessiot [12] who however does not seem to have written out a proof of the correctness of his algorithm. This is provided by Theorem 4.2 which is a general proof of correctness and supplies the above mentioned parametrisation as a corollary. First however, let us notice that the system consisting of equations (3.7) and (3.8) do indeed constitue the $A_{2}$ Toda field theory discussed in Leznov and Saveliev [8].

The equations (3.7) and (3.8) have the form

$$
\begin{aligned}
& \frac{\partial^{2}}{\partial x_{1} \partial x_{2}} \ln |h|=2 h-k \\
& \frac{\partial^{2}}{\partial x_{1} \partial x_{2}} \ln |k|=-h+2 k
\end{aligned}
$$


where we have abbreviated $h(L)$ to $h$ and $k(L)$ to $k$. Now suppose there exists an open subset of $\mathbb{R}^{2}$ such that $h k>0$. Then in this subset there exists functions $u\left(x_{1}, x_{2}\right)$ and $v\left(x_{1}, x_{2}\right)$ such that $u$ and $v$ satisfy the system

$$
\begin{gathered}
\frac{\partial^{2} u}{\partial x_{1} \partial x_{2}}=2 e^{u}-e^{v}, \\
\frac{\partial^{2} v}{\partial x_{1} \partial x_{2}}=-e^{u}+2 e^{v} .
\end{gathered}
$$

System (3.11) is precisely the Toda field theory for $A_{2}$ and corresponds to the Leznov-Saveliev system (1.1) when

$$
k=\left(\begin{array}{cc}
2 & -1 \\
-1 & 2
\end{array}\right)
$$

which is the Cartan matrix for the Lie algebra $A_{2}$. A further system is obtained according to the existence of open subsets of $\mathbb{R}^{2}$ in which $h k<0$. In this case functions $u$ and $v$ exist such that

$$
\begin{aligned}
& \frac{\partial^{2} u}{\partial x_{1} \partial x_{2}}=-2 e^{u}-e^{v}, \\
& \frac{\partial^{2} v}{\partial x_{1} \partial x_{2}}=e^{u}+2 e^{v} .
\end{aligned}
$$

Clearly (3.12) is equivalent to (3.11) with the change of variable $v \rightarrow v+i \pi$ but it is not clear that there exists a real local diffeomorphism relating these systems. The system (3.12) is not related to the Cartan matrix of any simple Lie algebra but is nevertheless an explicitly integrable system as we shall see.

\section{A LOCAL EQUiVALENCE PROBLEM FOR VECTOR FIELD SYSTEMS AND INTEgRATION OF THE $\boldsymbol{A}_{2}$ TODA FIELD THEORY}

In the previous section we showed that when the conditions defining the $(2,2)-$ Darboux integrability of the operators $L \in \mathcal{D}$ are expressed in terms of the Laplace invariants of $L$ one recovers the $A_{2}$ Toda field theory. By combining this result with the solution of a local equivalence problem for vector field systems (Theorem 4.2) we obtain in this section an explicit solution of the Toda field theory containing four (almost) arbitrary functions. (The word "almost" in the previous sentence will be explained in the sequel.) Here we understand by a "solution of an equivalence problem", the construction of an explicit diffeomorphism which maps one vector field system to another. This should be contrasted with the solution of a Cartan equivalence problem which employs powerful techniques to find necessary and sufficient conditions for equivalence in an algorithmic way in the form of a complete set of invariants [3]. 
Recall that $\Gamma_{2}(2,2)$ is the set of modules of Monge characteristic vector fields associated with the (2,2)-Darboux integrable partial differential equations

$$
\frac{\partial^{2} u}{\partial x_{1} \partial x_{2}}=f\left(x_{1}, x_{2}, u, \frac{\partial u}{\partial x_{1}}, \frac{\partial u}{\partial x_{2}}\right) .
$$

Call this set of equations $E(2,2)$. That is, $f \in E(2,2)$ if and only if $\tilde{\mu}(f) \in$ $\Gamma_{2}(2,2)$. Vessiot [12], has proved the following remarkable theorem regarding the set of equations $E(2,2)$.

ThEOREM 4.1 (VESSIOT). Let $\Delta_{2}(f) \subset J^{2}\left(\mathbb{R}^{2}, \mathbb{R}\right)$ denote the submanifold in the second order jet bundle defined by a partial differential equation $f \in E(2,2)$.

Then there exist

1. functions $\phi_{\alpha}, \psi_{\alpha} \in C^{\infty}(\mathbb{R}, \mathbb{R}), \alpha=1,2,3$;

2. a Lie group $G$, with $\operatorname{dim} G=3$;

3. a local diffeomorphism $\pi: \Delta_{2}(f) \rightarrow G \times \mathbb{R}^{4}$ such that

$$
\begin{aligned}
& \pi_{\star}\left({ }_{1} \Omega^{(2)}(f)\right)=\left\{\frac{\partial}{\partial u}+u_{1} \phi_{\alpha}(u) L_{\alpha}, \frac{\partial}{\partial u_{1}}\right\} \\
& \pi_{\star}\left({ }_{2} \Omega^{(2)}(f)\right)=\left\{\frac{\partial}{\partial v}+v_{1} \psi(v) R_{\alpha}, \frac{\partial}{\partial v_{1}}\right\}
\end{aligned}
$$

where $\left\{L_{\alpha}\right\}_{\alpha=1}^{3}$ and $\left\{R_{\alpha}\right\}_{\alpha=1}^{3}$ form bases for the left and right invariant vector fields on $G$ respectively. Finally, $\left\{u, u_{1}\right\}$ are any two functionally independent invariants of of ${ }_{2} \Omega^{(2)}(f)$ while $\left\{v, v_{1}\right\}$ are any two functionally independent invariants of ${ }_{1} \Omega^{(2)}(f)$.

Vessiot's theorem gives us a canonical form for the collection of modules

$$
\tilde{\mu}(E(2,2))={ }_{1} \Omega^{(2)}(f) \oplus{ }_{2} \Omega^{(2)}(f), \quad f \in E(2,2) .
$$

in terms of the three dimensional Lie groups. However the problem that we are interested in here may be thought of as the "converse" of Vessiot's theorem. That is, given a module of vector fields on $\mathbb{R}^{4} \times G$ of the form

$$
\left\{\frac{\partial}{\partial u}+u_{1} \phi_{\alpha}(u) L_{\alpha}, \frac{\partial}{\partial u_{1}}\right\} \oplus\left\{\frac{\partial}{\partial v}+v_{1} \psi_{\alpha}(v) R_{\alpha}, \frac{\partial}{\partial v_{1}}\right\},
$$

find the inverse of Vessiot's local diffeomorphism $\pi$. Once $\pi^{-1}$ has been found one can recover the associated partial differential equation $f \in E(2,2)$. As a corollary of this we integrate the Toda equations by a suitable choice of Lie group $G$. We note in passing that Vessiot's theorem is an existence result and the theorem itself gives no 
clue as to how $\pi$ or its inverse can be constructed. Nevertheless Vessiot's paper does contain clues and explicit computations; however there is no proof. This is provided by the following theorem.

Let us denote the collection of modules of vector fields of the form (4.1) by $\Sigma\left(\phi_{\alpha} ; \psi_{\alpha} ; G\right)$, which is shorthand for $\Sigma\left(\left\{\phi_{\alpha}\right\}_{\alpha=1}^{3} ;\left\{\psi_{\alpha}\right\}_{\alpha=1}^{3} ; G\right)$.

THEOREM 4.2. Suppose $\sigma \in \Sigma\left(\phi_{\alpha} ; \psi_{\alpha} ; G\right)$ is a module of vector fields on $\mathbb{R}^{4} \times G$ generated by

$$
\begin{aligned}
\sigma & =\left\{\frac{\partial}{\partial u}+u_{1} \phi_{\alpha}(u) L_{\alpha}, \frac{\partial}{\partial u_{1}}\right\} \oplus\left\{\frac{\partial}{\partial v}+v_{1} \psi_{\alpha} R_{\alpha}, \frac{\partial}{\partial v_{1}}\right\} \\
& =\left\{\mathbf{L}, \frac{\partial}{\partial u_{1}}\right\} \oplus\left\{\mathbf{R}, \frac{\partial}{\partial v_{1}}\right\}
\end{aligned}
$$

Suppose the vector field system $\mathcal{A}$ generated by

$$
\mathcal{A}=\left\{\left[\frac{\partial}{\partial u_{1}}, \mathbf{L}\right],\left[\frac{\partial}{\partial v_{1}}, \mathbf{R}\right], \frac{\partial}{\partial u_{1}}, \frac{\partial}{\partial v_{1}}\right\} \subset \sigma \oplus[\sigma, \sigma],
$$

has three functionally independent invariants $x_{1}, x_{2}$, and $z$ such that $\mathbf{L} x_{2}=\mathbf{R} x_{1}=0$ and $\mathbf{L} x_{1} \mathbf{R} x_{2} \neq 0$.

Define the functions

$$
z_{1}=\frac{\mathbf{L} z}{\mathbf{L} x_{1}}, z_{2}=\frac{\mathbf{R} z}{\mathbf{R} x_{1}}, \quad z_{11}=\frac{\mathbf{L} z_{1}}{\mathbf{L} x_{1}}, z_{22}=\frac{\mathbf{R} z_{2}}{\mathbf{R} x_{2}} .
$$

Then whenever the functions $\left(x_{1}, x_{2}, z, z_{1}, z_{2}, z_{11}, z_{22}\right)$ are functionally independent they define a local diffeomorphism

$$
\mathcal{P}: \mathbb{R}^{4} \times G \rightarrow \Delta_{2}(f)
$$

such that,

$$
\mathcal{P}_{\star}(\sigma)={ }_{1} \Omega^{(2)}(f) \oplus{ }_{2} \Omega^{(2)}(f),
$$

where $f$ is defined by

$$
z_{12}=\frac{\mathbf{L} z_{2}}{\mathrm{~L} x_{1}}=\frac{\mathbf{R} z_{1}}{\mathbf{R} x_{2}} .
$$

Furthermore, $f \in E(2,2)$ whenever $\frac{\partial f}{\partial z_{11}}=\frac{\partial f}{\partial z_{22}}=0$.

This theorem says that if the stated conditions are satisfied for $\sigma \in \Sigma\left(\phi_{\alpha} ; \psi_{\alpha} ; G\right)$ then a local diffeomorphism $\mathcal{P}: \mathbb{R}^{4} \times G \rightarrow \Delta_{2}(f)$ can be explicitly constructed such that

$$
\mathcal{P}_{\star}(\sigma) \in \Gamma_{2}(2,2)
$$


It then follows that $\mathcal{P}$ is the sought after inverse of Vessiot's diffeomorphism $\pi$. Moreover, we shall see that if we choose $\sigma$ from the collection $\Sigma\left(\phi_{\alpha} ; \psi_{\alpha} ; \mathbb{R}^{3}\right)$ of modules of vector field systems then $\mathcal{P}_{\star}(\sigma) \in \gamma_{2}(2,2)$, the image under $\mu$ of the $(2,2)$-Darboux integrable linear differential operators in $\mathcal{D}$.

ProOF of THEOREM 4.2: The proof proceeds as in [10] by finding a particular solution of the partial differential equations which define the equivalence map:

$$
\mathcal{P}: \mathbb{R}^{4} \times G \rightarrow \Delta_{2}(f)
$$

such that

$$
\mathcal{P}_{\star}(\sigma)={ }_{1} \Omega^{(2)}(f) \oplus_{2} \Omega^{(2)}(f)
$$

Such an equivalence implies the existence of a function $g: \mathbb{R}^{4} \times G \rightarrow G L(4, \mathbb{R})$ such that

$$
\mathcal{P}_{\star}\left(\begin{array}{c}
\mathbf{L} \\
\mathbf{R} \\
\frac{\partial}{\partial u_{1}} \\
\frac{\theta}{\partial v_{1}}
\end{array}\right)=g\left(\begin{array}{c}
D_{1}^{(2)} \\
D_{2}^{(2)} \\
\frac{\theta}{\partial z_{11}} \\
\frac{\theta}{\partial z_{22}}
\end{array}\right),
$$

where $D_{1}^{(2)}, D_{2}^{(2)}, \frac{\partial}{\partial z_{11}}$ and $\frac{\partial}{\partial z_{22}}$ are generators of ${ }_{1} \Omega^{(2)}(f) \oplus_{2} \Omega^{(2)}(f)$ for some partial differential equation

$$
\frac{\partial^{2} y}{\partial x_{1} \partial x_{2}}=f\left(x_{1}, x_{2}, y, \frac{\partial y}{\partial x_{1}}, \frac{\partial y}{\partial x_{2}}\right) .
$$

Since it is enough to find a particular solution of the overdetermined system of first order partial differential equations defining the equivalence map $\mathcal{P}$, we specialise $g$ to lie in the subgroup of $G L(4, \mathbb{R})$ described by the matrices

$$
g=\left(\begin{array}{cccc}
\omega_{11} & 0 & \omega_{13} & \omega_{14} \\
0 & \omega_{22} & \omega_{23} & \omega_{24} \\
0 & 0 & \omega_{33} & \omega_{34} \\
0 & 0 & \omega_{43} & \omega_{44}
\end{array}\right)
$$

This choice of subgroup is based purely on heuristic arguments. Hence,

$$
\begin{gathered}
\mathcal{P}_{\star}(\mathrm{L})=\omega_{11} D_{1}^{(2)}+\omega_{13} \frac{\partial}{\partial z_{11}}+\omega_{14} \frac{\partial}{\partial z_{22}}, \mathcal{P}_{\star}(\mathbf{R})=\omega_{22} D_{2}^{(2)}+\omega_{23} \frac{\partial}{\partial z_{11}}+\omega_{24} \frac{\partial}{\partial z_{22}} \\
\mathcal{P}_{\star}\left(\frac{\partial}{\partial u_{1}}\right)=\omega_{33} \frac{\partial}{\partial z_{11}}+\omega_{34} \frac{\partial}{\partial z_{22}}, \mathcal{P}_{\star}\left(\frac{\partial}{\partial v_{1}}\right)=\omega_{43} \frac{\partial}{\partial z_{11}}+\omega_{44} \frac{\partial}{\partial z_{22}}
\end{gathered}
$$

Note that $\Delta_{2}(f)$ has local coordinates $x_{1}, x_{2}, z, z_{1}, z_{2}, z_{11}, z_{22}$, that is, all the local coordinates of $J^{2}\left(\mathbb{R}^{2}, \mathbb{R}\right)$ except for $z_{12}$ since this coordinate is specified by the partial differential equation $f$. 
The local coordinate expressions for $D_{1}^{(2)}$ and $D_{2}^{(2)}$ when combined with equations (4.2) give the following overdetermined system for $\mathcal{P}$ :

$$
\begin{aligned}
\mathbf{L} x_{1} & =\omega_{11}, \frac{\partial x_{1}}{\partial u_{1}}=0, \frac{\partial x_{1}}{\partial v_{1}}=0, \mathbf{R} x_{1}=0 \\
\mathbf{R} x_{2} & =\omega_{22}, \frac{\partial x_{2}}{\partial u_{1}}=0, \frac{\partial x_{2}}{\partial v_{1}}=0, \mathbf{L} x_{2}=0 \\
\mathbf{L} z & =\omega_{11} z_{1}, \frac{\partial z}{\partial u_{1}}=0, \frac{\partial z}{\partial v_{1}}=0, \mathbf{R} z=\omega_{22} z_{2} \\
\mathbf{L} z_{1} & =\omega_{11} z_{11}, \mathbf{R} z_{1}=\omega_{22} f, \frac{\partial z_{1}}{\partial u_{1}}=0, \frac{\partial z_{1}}{\partial v_{1}}=0 \\
\mathbf{L} z_{2} & =\omega_{11} f, \mathbf{R} z_{2}=\omega_{22} z_{22}, \frac{\partial z_{1}}{\partial u_{1}}=0, \frac{\partial z_{2}}{\partial v_{1}}=0 \\
\mathbf{L} z_{11} & =\omega_{13}, \mathbf{R}=\omega_{23}, \frac{\partial z_{11}}{\partial u_{1}}=0, \frac{\partial z_{11}}{\partial v_{1}}=0 \\
\mathbf{L} z_{22} & =\omega_{14}, \mathbf{R} z_{22}=\omega_{24}, \frac{\partial z_{22}}{\partial u_{1}}=\omega_{34}, \frac{\partial z_{22}}{\partial v_{1}}=\omega_{44}
\end{aligned}
$$

Suppose for the present that we can find $x_{1}$ and $x_{2}$ which satisfy $(4.3)_{1}$ and $(4.3)_{2}$. Let us consider the equation (4.3) ${ }_{3}$. From the first two equations we have

$$
\left[\frac{\partial}{\partial u_{1}}, \mathbf{L}\right] z=\frac{\partial}{\partial u_{1}}(\mathbf{L} z)-\mathbf{L}\left(\frac{\partial z}{\partial u_{1}}\right)=\frac{\partial}{\partial u_{1}}\left(\omega_{11} z_{1}\right)=\frac{\partial \omega_{11}}{\partial u_{1}} z_{1}+\omega_{11} \frac{\partial z_{1}}{\partial u_{1}}
$$

and from the second two equations we obtain

$$
\left[\frac{\partial}{\partial v_{1}}, \mathbf{R}\right] z=\frac{\partial \omega_{22}}{\partial v_{1}} z_{2}+\omega_{22} \frac{\partial z_{2}}{\partial v_{1}}
$$

But from $(4.3)_{1}$ and $(4.3)_{2}$ we find that

$$
\frac{\partial \omega_{11}}{\partial u_{1}}=\left[\frac{\partial}{\partial u_{1}}, \mathbf{L}\right] x_{1}, \frac{\partial \omega_{22}}{\partial v_{1}}=\left[\frac{\partial}{\partial v_{1}}, \mathbf{R}\right] x_{2}
$$

Suppose then that at this point, in addition to $\mathbf{R} x_{1}=\mathbf{L} x_{2}=0$ we require that $x_{1}$ and $x_{2}$ be invariants of the vector field system

$$
\left\{\frac{\partial}{\partial u_{1}}, \frac{\partial}{\partial v_{1}},\left[\frac{\partial}{\partial u_{1}}, \mathbf{L}\right],\left[\frac{\partial}{\partial v_{1}}, \mathbf{R}\right]\right\}
$$

such that $\left(\mathbf{L} x_{1}\right)\left(\mathbf{R} x_{2}\right) \neq 0$.

Matters being so, equations (4.4) and (4.5) become

$$
\frac{\partial z_{1}}{\partial u_{1}}=\left(\omega_{11}\right)^{-1}\left[\frac{\partial}{\partial u_{1}}, \mathbf{L}\right] z, \frac{\partial z_{2}}{\partial v_{1}}=\left(\omega_{22}\right)^{-1}\left[\frac{\partial}{\partial v_{1}}, \mathbf{R}\right] z .
$$


In addition we have

$$
\frac{\partial z_{1}}{\partial v_{1}}=\left(\omega_{11}\right)^{-1}\left[\frac{\partial}{\partial v_{1}}, \mathbf{L}\right] z, \frac{\partial z_{2}}{\partial u_{1}}=\left(\omega_{22}\right)^{-1}\left[\frac{\partial}{\partial u_{1}}, \mathbf{R}\right] .
$$

Thus in order to satisfy the middle two equations of $(4.3)_{3}$, the third equation in $(4.3)_{4}$ and the last equation in $(4.3)_{5}$ we need to require that $z$ be an invariant of (4.7). From (4.9) we see that $\frac{\partial z_{1}}{\partial u_{1}}=\frac{\partial z_{2}}{\partial v_{1}}=0$ are satisfied by virtue of the fact that $\left[\frac{\partial}{\partial v_{1}}, \mathbf{L}\right]=\left[\frac{\partial}{\partial u_{1}}, \mathbf{R}\right]=0$. This effectively solves the partial differential equations defining the equivalence map $\mathcal{P}$.

Summarising, we have shown that if $x_{1}, x_{2}$ and $z$ are chosen to be any functionally independent invariants of

$$
\left\{\frac{\partial}{\partial u_{1}}, \frac{\partial}{\partial v_{1}},\left[\frac{\partial}{\partial u_{1}}, \mathbf{L}\right],\left[\frac{\partial}{\partial v_{1}}, \mathbf{R}\right]\right\}
$$

such that $\left(\mathbf{L} x_{1}\right)\left(\mathbf{R} x_{2}\right) \neq 0$ and $\mathbf{R} x_{1}=\mathbf{L} x_{2}=0$ then the remaining variables are obtained from the system $(4.3)_{3}$ to $(4.3)_{5}$ as in the statement of the theorem. In particular the partial differential equation $f$ is defined by

$$
f=\frac{\mathbf{L} z_{2}}{\mathbf{L} x_{2}}=\frac{\mathbf{R} z_{1}}{\mathbf{R} x_{2}}
$$

and justifies our assertion that $\mathcal{P}$ satisfies

$$
\mathcal{P}_{\star}(\sigma)={ }_{1} \Omega^{(2)}(f) \oplus{ }_{2} \Omega^{(2)}(f),
$$

for some partial differential equation

$$
\frac{\partial^{2} y}{\partial x_{1} \partial x_{2}}=f\left(x_{1}, x_{2}, y, \frac{\partial y}{\partial x_{1}}, \frac{\partial y}{\partial x_{2}}, \frac{\partial^{2} y}{\partial x_{1}^{2}}, \frac{\partial^{2} y}{\partial x_{2}^{2}}\right)
$$

We must now show that if $\frac{\partial f}{\partial z_{11}}=\frac{\partial f}{\partial z_{22}}=0$ then $\tilde{\mu}(f) \in E(2,2)$. First of all we show that with $\mathcal{P}$ defined as in the statement of the Theorem, then $\mathcal{P}_{\star}$ maps $\left\{\mathrm{L}, \frac{\partial}{\partial u_{1}}\right\}$ to ${ }_{1} \Omega^{(2)}(f)$ and $\left\{\mathbf{R}, \frac{\partial}{\partial v_{1}}\right\}$ to ${ }_{2} \Omega^{(2)}(f)$. To do this we need to first show that $\omega_{34}=\omega_{43}=0$, since then we have

$$
\mathcal{P}_{\star}\left(\frac{\partial}{\partial u_{1}}\right)=\omega_{33} \frac{\partial}{\partial z_{11}}, \mathcal{P}_{\star}\left(\frac{\partial}{\partial v_{1}}\right)=\omega_{44} \frac{\partial}{\partial z_{22}}
$$

Now $\omega_{34}=\frac{\partial z_{22}}{\partial u_{1}}$ and from the equations $\mathbf{R} z_{2}=\omega_{22} z_{22}$ and $\frac{\partial z_{2}}{\partial u_{1}}=0$ we have

$$
\frac{\partial}{\partial u_{1}}\left(\mathbf{R} z_{2}\right)-\mathbf{R} \frac{\partial z_{2}}{\partial u_{1}}=\left[\frac{\partial}{\partial u_{1}}, \mathbf{R}\right] z_{2}=\frac{\partial \omega_{22}}{\partial u_{1}} z_{22}+\omega_{22} \frac{\partial z_{22}}{\partial u_{1}}
$$


However with $\mathcal{P}$ defined as above we have $\frac{\partial \omega_{22}}{\partial u_{1}}=0$, whence

$$
\frac{\partial z_{22}}{\partial u_{1}}=\omega_{22}^{-1}\left[\frac{\partial}{\partial u_{1}}, \mathbf{R}\right] z_{2}
$$

Noting that $\left[\frac{\partial}{\partial u_{1}}, \mathbf{R}\right]=0$ gives the required result. A similar argument shows that

$$
\frac{\partial z_{11}}{\partial v_{1}}=\omega_{11}^{-1}\left[\frac{\partial}{\partial v_{1}}, \mathrm{~L}\right] z_{1}
$$

and since $\left[\frac{\partial}{\partial v_{1}}, \mathrm{~L}\right]=0$ the result follows.

So far we have shown that

$$
\begin{aligned}
& \mathcal{P}_{\star}\left\{\mathrm{L}, \frac{\partial}{\partial u_{1}}\right\}=\left\{D_{1}^{(2)}+\frac{\omega_{14}}{\omega_{11}} \frac{\partial}{\partial z_{22}}, \frac{\partial}{\partial z_{11}}\right\}, \\
& \mathcal{P}_{\star}\left\{\mathbf{R}, \frac{\partial}{\partial v_{1}}\right\}=\left\{D_{2}^{(2)}+\frac{\omega_{23}}{\omega_{22}} \frac{\partial}{\partial z_{11}}, \frac{\partial}{\partial z_{22}}\right\}
\end{aligned}
$$

Our next job is to show that

$$
\frac{\omega_{14}}{\omega_{11}}=D_{2}^{(2)}(f) \text { and } \frac{\omega_{23}}{\omega_{22}}=D_{1}^{(2)}(f)
$$

To do this we first generalise the well known fact that if $F=\left\{X_{1}, X_{2}, \ldots, X_{m}\right\}$ is a $C^{\infty}\left(\mathbb{R}^{n}, \mathbb{R}\right)$ module of vector fields of constant dimension on an open subset $U$ of $\mathbb{R}^{n}$ and if $\phi: U \rightarrow U$ is a local diffeomorphism then $\phi_{\star}(\operatorname{char} F)=\operatorname{char}\left(\phi_{\star} F\right)$. That is, characteristic submodules are maped to characteristic submodules. Indeed for fixed $X \in F$, write

$$
F_{X}^{(1)}=F \oplus[X, F]
$$

We assume that here as elsewhere we are in the nonsingular case where the dimension of all vector field systems "derived" from $F$ does not change on $U$. Following Stormark [9], we define a function

by

$$
r_{F}: F \rightarrow \mathbb{R}
$$

Let

$$
\begin{gathered}
r_{F}(X)=\operatorname{dim}\left(F_{X}^{(1)} / F\right) . \\
r_{1}=\max _{X \in F} r_{F}(X)
\end{gathered}
$$

Call $X$ regular in $F$ if $r_{F}(X)=r_{1}$; singular in $F$ if $r_{F}(X)<r_{1}$ and characteristic in $F$ if $r_{F}(X)=0$. The following is easy to establish. 
LEMMA. $r_{F}$ is a $\operatorname{Diff}_{\text {loc }}\left(\mathbb{R}^{n}\right)$ invariant.

This result generalises the well known fact that if $X$ is characteristic in $F$ then $\phi_{\star} X$ is characteristic in $\phi_{\star} F$. So if we give the number $r_{F}(X)$ the obvious name of the rank of $X$ in $F$ then we see that it is a $\operatorname{Diff}$ loc $\left(\mathbb{R}^{n}\right)$ invariant so that $X$ being characteristic corresponds to the special case $r_{F}(X)=0$. More generally, regular vector fields are maped to regular vector fields and singular to singular.

\section{LEMMA .}

The local diffeomorphism $\mathcal{P}$, when acting on $\left\{\mathbf{L}, \frac{\partial}{\partial u_{1}}\right\}$ and $\left\{\mathbf{R}, \frac{\partial}{\partial v_{1}}\right\}$ maps these to the Monge characteristic vector field systems. That is,

and

$$
\begin{aligned}
& \mathcal{P}_{\star}\left\{\mathbf{L}, \frac{\partial}{\partial u_{1}}\right\}={ }_{1} \Omega^{2}(f), \\
& \mathcal{P}_{\star}\left\{\mathbf{R}, \frac{\partial}{\partial v_{1}}\right\}={ }_{2} \Omega^{2}(f),
\end{aligned}
$$

for some $f \in E(2,2)$.

Proof: We prove the result for $\left\{\mathrm{L}, \frac{\partial}{\partial u_{1}}\right\}$; the other being similar. Firstly, we note that

$$
\sigma_{\mathbf{L}}^{(1)} / \sigma=\phi_{\alpha} L_{\alpha}
$$

Assuming that not all the $\phi_{\alpha}$ are zero, we have $r_{\sigma}(L)=1$. Let us compute

$$
\mathcal{H}=\left[\mathcal{P}_{\star} \mathrm{L}, \mathcal{P}_{\star} \sigma\right] / \mathcal{P}_{\star} \sigma
$$

We have already shown that

$$
\mathcal{P}_{\star} \mathrm{L}=\omega_{11} D_{1}^{(2)}+\omega_{13} \frac{\partial}{\partial z_{11}}+\omega_{14} \frac{\partial}{\partial z_{14}},
$$

and by construction that,

$$
\mathcal{P}_{\star} \sigma=\left\{D_{1}^{(2)}, D_{2}^{(2)}, \frac{\partial}{\partial z_{11}}, \frac{\partial}{\partial z_{22}}\right\}
$$

where,

$$
\begin{aligned}
& D_{1}^{(2)}=\frac{\partial}{\partial x_{1}}+z_{1} \frac{\partial}{\partial z}+z_{11} \frac{\partial}{\partial z_{1}}+f \frac{\partial}{\partial z_{2}} \\
& D_{2}^{(2)}=\frac{\partial}{\partial x_{2}}+z_{2} \frac{\partial}{\partial z}+f \frac{\partial}{\partial z_{1}}+z_{22} \frac{\partial}{\partial z_{2}}
\end{aligned}
$$

are the total differential operators restricted to $\Delta_{2}(f)$. 
Calculation then gives

$$
\begin{aligned}
& {\left[D_{1}^{(2)}, \mathcal{P}_{\star} \mathbf{L}\right] \equiv \omega_{14} \frac{\partial}{\partial z_{1}},\left[\frac{\partial}{\partial z_{11}}, \mathcal{P}_{\star} \mathbf{L}\right] \equiv \omega_{11} \frac{\partial}{\partial z_{1}}} \\
& {\left[D_{2}^{(2)}, \mathcal{P}_{\star} \mathbf{L}\right] \equiv \omega_{11}\left(\left(D_{1}^{(2)} f\right) \frac{\partial}{\partial z_{1}}-\left(D_{2}^{(2)} f\right) \frac{\partial}{\partial z_{2}}\right)+\omega_{14} \frac{\partial}{\partial z_{2}},\left[\frac{\partial}{\partial z_{22}}, \mathcal{P}_{\star} \mathbf{L}\right] \equiv 0 .}
\end{aligned}
$$

Hence

$$
\mathcal{H}=\left\{\frac{\partial}{\partial z_{1}},\left(\omega_{14}-\omega_{11} D_{2}^{(2)} f\right) \frac{\partial}{\partial z_{2}}\right\} .
$$

Now by the previous lemma, $r_{\mathcal{P}_{\star} \sigma}\left(\mathcal{P}_{\star} \mathrm{L}\right)=1$ since $\mathcal{P}$ is a local diffeomorphism. Hence $\mathcal{H}$ must have dimension one. This in turn forces $\omega_{14}-\omega_{11} D_{2}^{(2)} f=0$.

Now, because

$$
\mathcal{P}_{\star}\left(\frac{\partial}{\partial u_{1}}\right)=\omega_{33} \frac{\partial}{\partial z_{11}},
$$

we have shown, upon comparing with Definition 3.1, that

$$
\mathcal{P}_{\star}\left\{\mathbf{L}, \frac{\partial}{\partial u_{1}}\right\}={ }_{1} \Omega^{(2)}(f) .
$$

A similar argument shows that

$$
\mathcal{P}_{\star}\left\{\mathbf{R}, \frac{\partial}{\partial v_{1}}\right\}={ }_{2} \Omega^{(2)}(f)
$$

Finally, we note that $\left\{\mathrm{L}, \frac{\partial}{\partial u_{1}}\right\}$ has two functionally independent invariants $\left(v, v_{1}\right)$ while $\left\{\mathbf{R}, \frac{\partial}{\partial v_{1}}\right\}$ has two functionally independent invariants $\left(u, u_{1}\right)$. It follows that their diffeomorphic images, ${ }_{1} \Omega^{(2)}(f)$ and ${ }_{2} \Omega^{(2)}(f)$, each have two functionally independent invariants. This proves that $f \in E(2,2)$ and completes the proof of the lemma and the theorem.

We are now in a position to solve the $A_{2}$ Toda field theory by making use of Theorem 4.2 and specialising the Lie group $G$ that appears there to be the vector space $\mathbb{R}^{3}$. That is, we study the collection of vector field systems $\Sigma\left(\phi_{\alpha} ; \psi_{\alpha} ; \mathbb{R}^{3}\right)$. Explicitly these vector field systems have the form

$$
\begin{aligned}
& \sigma=\left\{\frac{\partial}{\partial u}+u_{1}\left(\phi_{1}(u) \frac{\partial}{\partial w_{1}}+\right.\right.\left.\phi_{2}(u) \frac{\partial}{\partial w_{2}}+\phi_{3}(u) \frac{\partial}{\partial w_{3}}\right), \frac{\partial}{\partial u_{1}} \\
&\left.\frac{\partial}{\partial v}+v_{1}\left(\psi_{1}(v) \frac{\partial}{\partial w_{1}}+\psi_{2}(v) \frac{\partial}{\partial w_{2}}+\psi_{3}(v) \frac{\partial}{\partial w_{3}}\right), \frac{\partial}{\partial v_{1}}\right\},
\end{aligned}
$$


where $w_{1}, w_{2}$ and $w_{3}$ are coordinates on $\mathbb{R}^{3}$. Now $\sigma$ is defined up to local diffeomorphisms so we may use this freedom to simplify $\sigma$. Indeed, by re-parametrising $u$ and $v$ we can transform $\phi_{1}$ and $\psi_{1}$ (say) to 1 . This means that we can without loss of generality study the family of modules $\Sigma\left(1, \phi_{2}, \phi_{3} ; 1, \psi_{2}, \psi_{3} ; \mathbb{R}^{3}\right)$. Now it transpires that the condition necessary and sufficient in order that the submodules $\left\{L, \frac{\partial}{\partial u_{1}}\right\}$ and $\left\{\mathbf{R}, \frac{\partial}{\partial v_{1}}\right\}$ each have two functionally independent invariants is that the Wronskians of $\left(1, \phi_{2}, \phi_{3}\right)$ and $\left(1, \psi_{2}, \psi_{3}\right)$ are each not identically zero. That is, the $\phi$ 's and $\psi$ 's must be linearly independent.

Matters being so, application of Theorem 4.2 suggests that we compute the invariants of the submodule $\mathcal{A}$ of $\sigma^{(1)}=\sigma \oplus[\sigma, \sigma]$. In this case $\mathcal{A}$ is given by

$$
\left\{\frac{\partial}{\partial w_{1}}+\phi_{2} \frac{\partial}{\partial w_{2}}+\phi_{3} \frac{\partial}{\partial w_{3}}, \frac{\partial}{\partial w_{1}}+\psi_{2} \frac{\partial}{\partial w_{2}}+\psi_{3} \frac{\partial}{\partial w_{3}}, \frac{\partial}{\partial u_{1}}, \frac{\partial}{\partial v_{1}}\right\}
$$

Under the stated nondegeneracy conditions on the $\phi$ 's and $\psi$ 's, $\mathcal{A}$ will have three functionally independent invariants which are easily computed:

where

$$
\begin{gathered}
x_{1}=u, x_{2}=v, z=w_{1} \theta_{1}+w_{2} \theta_{2}+w_{3} \theta_{3}, \\
\theta_{1}=\phi_{2} \psi_{3}-\phi_{2} \psi_{3}, \theta_{2}=\phi_{3}-\psi_{3}, \theta_{3}=\psi_{2}-\phi_{3} .
\end{gathered}
$$

These invariants satisfy the conditions of Theorem 4.2 and define a local diffeomorphism, as may easily be checked directly. Now in order to construct the partial differential equations associated with any $\sigma \in \Sigma\left(1, \phi_{2}, \phi_{3} ; 1, \psi_{2}, \psi_{3} ; \mathbb{R}^{3}\right)$ we need to compute $z_{1}, z_{2}$ and $z_{12}$. By applying Theorem 4.2 once again we have (only by carrying out differentiations)

$$
\begin{aligned}
z_{1} & =\sum_{\alpha=1}^{s} w_{\alpha} \frac{\partial \theta_{\alpha}}{\partial u}, z_{2}=\sum_{\alpha=1}^{3} w_{\alpha} \frac{\partial \theta_{\alpha}}{\partial v} \\
z_{12} & =\sum_{\alpha=1}^{3} w_{\alpha} \frac{\partial^{2} \theta_{\alpha}}{\partial u \partial v}=w_{1} \frac{\partial^{2} \theta_{1}}{\partial u \partial v} .
\end{aligned}
$$

We are therefore led naturally to express $w_{1}$ as a function of the local co-ordinates on $\Delta_{2}(f)$. Combining (4.10) with (4.11) we find that $w_{1}, w_{2}$ and $w_{3}$ satisfy the linear algebraic system

$$
\begin{aligned}
z & =w_{1} \theta_{1}+w_{2} \theta_{2}+w_{3} \theta_{3} \\
z_{1} & =w_{1} \frac{\partial \theta_{1}}{\partial u}+w_{2} \frac{\partial \theta_{2}}{\partial u}+w_{3} \frac{\partial \theta_{3}}{\partial u} \\
z_{2} & =w_{1} \frac{\partial \theta_{1}}{\partial v}+w_{2} \frac{\partial \theta_{2}}{\partial v}+w_{3} \frac{\partial \theta_{3}}{\partial v}
\end{aligned}
$$


Because of the form of the $\phi$ 's and $\psi$ 's the above system can always be solved to express $w_{1}, w_{2}$ and $w_{3}$ as linear combinations of $z, z_{1}$ and $z_{2}$. Suppose we write the solution $w_{1}$ of (4.13) in the form

$$
w_{1}=A(u, v) z+B(u, v) z_{1}+C(u, v) z_{2}
$$

This then gives $\Delta_{2}(f)$ as a submanifold of $J^{2}\left(\mathbb{R}^{2}, \mathbb{R}\right)$ defined by

$$
z_{12}=\frac{\partial^{2} \theta_{1}}{\partial x_{1} \partial x_{2}}\left(A\left(x_{1}, x_{2}\right) z+B\left(x_{1}, x_{2}\right) z_{1}+C\left(x_{1}, x_{2}\right) z_{2}\right)
$$

and shows that the class of partial differential equations associated with $\Sigma\left(1, \phi_{2}, \phi_{3}\right.$; $1, \psi_{2}, \psi_{3} ; \mathbb{R}^{3}$ ) is parametrised by

$$
\frac{\partial^{2} y}{\partial x_{1} \partial x_{2}}=\frac{\partial^{2} \theta_{1}}{\partial x_{1} \partial x_{2}}\left(A\left(x_{1}, x_{2}\right) y+B\left(x_{1}, x_{2}\right) \frac{\partial y}{\partial x_{1}}+C\left(x_{1}, x_{2}\right) \frac{\partial y}{\partial x_{2}}\right)
$$

where $\theta_{1}, A, B$, and $C$ are defined as above. Since the partial differential equation (4.14) is independent of the higher order derivatives $\frac{\partial^{2} y}{\partial x_{1}^{2}}$ and $\frac{\partial^{2} y}{\partial x_{2}^{2}}$ it follows once again by Theorem 4.2 that the class of partial differential equations defined by (4.14) satisfies the condition that each member is $(2,2)$-Darboux integrable. In other words, we have explicitly constructed Vessiot's class of second order linear differential operators $\mu^{-1}\left(\gamma_{2}(2,2)\right)$, namely,

$$
L=\partial_{x_{1} x_{2}}^{2}+\frac{\partial^{2} \theta_{1}}{\partial x_{1} \partial x_{2}}\left(B \partial_{x_{1}}+C \partial_{x_{2}}+A\right)
$$

and hence $h(L)$ and $k(L)$ are by Theorem 3.4 solutions of equations (3.10). We thus obtain solutions of the Toda field equations containing four arbitrary linearly independent, twice differentiable functions each of one variable in the case when $h(L) k(L)>0$ and of equations (3.12) when $h(L) k(L)<0$.

EXAMPLE. We end by giving an elementary example to illustrate the theory. We construct the partial differential equation associated with the vector field system $\Sigma\left(1, u, u^{-1} ; 1, v, v^{-1} ; \mathbb{R}^{3}\right)$. In this case $\theta_{1}=u / v-v / u, \theta_{2}=1 / u-1 / v, \theta_{3}=v-u$ and 
we find that solving the linear algebraic system (4.13) leads to

$$
w_{1}=\frac{(u v)^{2}\left(u^{2}-v^{2}\right) z-u^{3} v^{2}(u-v)^{2} z_{1}+u^{2} v^{3}(u-v)^{2} z_{2}}{-u v(u-v)^{4}} .
$$

Then from

$$
z_{12}=\frac{\partial^{2} \theta_{1}}{\partial u \partial v} w_{1}
$$

one obtains the associated partial differential equation in the form

$$
\frac{\partial^{2} y}{\partial x_{1} \partial x_{2}}+\frac{\left(x_{1}+x_{2}\right)}{x_{2}\left(x_{1}-x_{2}\right)} \frac{\partial y}{\partial x_{1}}-\frac{\left(x_{1}+x_{2}\right)}{x_{1}\left(x_{1}-x_{2}\right)} \frac{\partial y}{\partial x_{2}}-\frac{\left(x_{1}+x_{2}\right)^{2}}{x_{1} x_{2}\left(x_{1}-x_{2}\right)^{2}} y=0
$$

The Laplace invariants of the operator defined by (4.15) are

$$
\begin{gathered}
h(L)=\frac{\partial}{\partial x_{1}}\left(\frac{x_{1}+x_{2}}{x_{2}\left(x_{1}-x_{2}\right)}\right)=\frac{-2}{\left(x_{1}-x_{2}\right)^{2}}, \\
k(L)=-\frac{\partial}{\partial x_{2}}\left(\frac{x_{1}+x_{2}}{x_{1}\left(x_{1}-x_{2}\right)}\right)=\frac{-2}{\left(x_{1}-x_{2}\right)^{2}} .
\end{gathered}
$$

Here we are in the "equal invariants" case because of the symmetric form we chose for the $\phi$ 's and $\psi$ 's. In this case the Toda equations degenerate to the Liouville equation for which (4.16) defines a solution. Obviously the choice of "nonsymmetric" forms for the $\phi$ 's and $\psi$ 's lead to other solutions of the Toda equations which are not solutions of the Liouville equation.

\section{REFERENCES}

[1] R. Bryant, S.S. Chern, R.B. Gardner, H. Goldschmidt and P. Grifths, Exterior differential systems, MSIR series (Springer-Verlag, Berlin, Heidelberg, New York, 1989).

[2] G. Darboux, Lecons sur la theorie generale de surface 2 (Gauthier-Villars, Paris, 1889).

[3] R.B. Gardner, The method of equivalence and its applications, SIAM CBMS - NSF Regional Conference Series, 1989.

[4] R.B. Gardner and N. Karman, 'Characteristics and the geometry of hyperbolic equations in the plane', J. Differential Equations (to appear).

[5] A.M. Grundland and P.J. Vassiliou, 'On the solvability of the Cauchy problem for Riemann double waves by the method of Monge - Darboux', Analysis 11 (1992), 221-278.

[6] A.M. Grundland and P.J. Vassiliou, 'Riemann double waves, Darboux method and the Painleve property', in Proccedings of the NATO workshop on Painleve transcendents, their asymptotics and physical applications, (D. Levi and P.Winternitz, Editors) (Plenum Press, 1992). 
[7] R. Hermann, The geometry of nonlinear differential equations, Interdisciplinary Mathematics 12, Backlund Transformations and Solitons Part A (Math Science Press, Brookline, MA., 1976).

[8] A.N. Leznov and M.V. Saveliev, 'Representation of zero curvature for the system of nonlinear partial differential equations $x_{\alpha, z \bar{z}}=\exp (k x)_{\alpha}$ and its integrability', Lett. Math. Phys. 3 (1979), 489-494.

[9] O. Stormark, 'Formal and local solvability of partial differential equations', Royal Swedish Institute of Technology (preprint).

[10] P.J. Vassiliou, 'On local equivalence for vector field systems', Bull. Austral. Math. Soc. 42 (1990), 215-229.

[11] P.J. Vassiliou, in preparation.

[12] E. Vessiot, 'Sur les equations aux derives partielles du second ordre $F(x, y, z, p, q, r, s, t)=0$ integrable par la methode de Darboux', J. Math. Pures Appl. 18 (1939), 1-61.ibid, 21 1-66, (1942) .

[13] J. Weiss, Backland transformations and the Painleve property, (preprint 509, April 1989) (Institute for Mathematics and its Applications, University of Minnesota).

Faculty of Information Sciences and Engineering

University of Canberra

Belconnen ACT 2616

Australia 